\title{
Síndrome do canal de Guyon por aneurisma da artéria ulnar: relato de caso
}

Guyon's canal syndrome due to a ulnar artery aneurysm: case report

Síndrome del canal de Guyon por aneurisma de la arteria cubital: reporte de caso

Luis Guilherme Rosifini Alves REZENDE ${ }^{\mathbf{1}}$

Letícia de Freitas LEONEL ${ }^{2}$

Filipe Jun SHIMAOKA ${ }^{1}$

Gunter Shin Iti Takamori SHIMABUKURO ${ }^{3}$

Luiz Garcia MANDARANO-FILHO ${ }^{1}$

Nilton MAZZER ${ }^{4}$

Cláudio Henrique BARBIERI ${ }^{4}$

${ }^{I}$ Cirurgião de Mão do Hospital das Clínicas da Faculdade de Medicina de Ribeirão Preto da Universidade de São Paulo (HC-FMRP-USP) 14015-010 Ribeirão Preto-SP, Brasil

${ }^{2}$ Residente em Ortopedia e Traumatologia no Hospital das Clínicas da Faculdade de Medicina de Ribeirão Preto da Universidade de São Paulo (HC-FMRP-USP) 14015-010 Ribeirão Preto - SP, Brasil

${ }^{3}$ Cirurgião de Mão do Hospital Estadual de Serrana, vinculado ao Complexo do Hospital das Clínicas da Faculdade de Medicina de Ribeirão Preto da Universidade de São Paulo (HC-FMRP-USP) 14015-010 Ribeirão Preto - SP, Brasil

${ }^{4}$ Professor Titular da Cirurgia da Mão do Hospital das Clínicas da Faculdade de Medicina de Ribeirão Preto da Universidade de São Paulo (HC-FMRP-USP) 14015-010 Ribeirão Preto - SP, Brasil

\section{Resumo}

Dentre os fatores que podem desencadear a compressão do canal de Guyon inclui-se o aneurisma da artéria ulnar. Uma das abordagens cirúrgicas é a reconstrução microcirúrgica através da ressecção e arteriorrafia término-terminal ou enxerto venoso de interposição. O objetivo deste trabalho é relatar o caso de paciente que desenvolveu neuropatia do nervo ulnar secundária à presença de aneurisma da artéria ulnar no canal de Guyon. Foi realizada a descompressão cirúrgica através de uma abertura do canal e a ressecção do segmento lesionado. A artéria ulnar foi reconstruída através da anastomose término-terminal microcirúrgica. O resultado foi o alívio completo dos sintomas no pósoperatório com manutenção do fluxo vascular.

Descritores: Nervo Ulnar; Neuropatia Radial; Ferimentos e Lesões; Aneurisma.

\section{Abstract}

Among the factors that can trigger the compression of the Guyon canal include the ulnar artery aneurysm. One of the surgical approaches is microsurgical reconstruction through resection and end-to-end arteriography or venous interposition graft. The objective of this study is to report the case of a patient who developed ulnar nerve neuropathy secondary to the presence of an ulnar artery aneurysm in the Guyon canal. Surgical decompression was performed through an opening of the canal and resection of the injured segment. The ulnar artery was reconstructed using microsurgical end-to-end anastomosis. The result was complete relief of symptoms in the postoperative period with maintenance of vascular flow.

Descriptors: Ulnar Nerve; Radial Neurophaty; Wounds and Injuries; Aneurysm.

\section{Resumen}

Entre los factores que pueden desencadenar la compresión del canal de Guyon se encuentran el aneurisma de la arteria cubital. Uno de los abordajes quirúrgicos es la reconstrucción microquirúrgica mediante resección y arteriografía término-terminal o injerto de interposición venosa. El objetivo de este estudio es reportar el caso de un paciente que desarrolló una neuropatía del nervio cubital secundario a la presencia de un aneurisma de la arteria cubital en el canal de Guyon. La descompresión quirúrgica se realizó mediante apertura del canal y resección del segmento lesionado. La arteria cubital se reconstruyó mediante anastomosis microquirúrgica de extremo a extremo. El resultado fue un alivio completo de los síntomas en el postoperatorio con mantenimiento del flujo vascular.

Descriptores: Nervio Cubital; Neuropatía Radial; Heridas y Traumatismos; Aneurisma.

\section{INTRODUÇÃO}

Desde a descrição do Canal de Guyon em 1861, diversas causas de compressão foram descritas, dentre elas, o aneurisma da artéria ulnar ${ }^{1-4}$. Existem diferentes formas de manejo cirúrgico, como a reconstrução microcirúrgica através da ressecção e arteriorrafia término-terminal ou enxerto venoso de interposição ${ }^{5,6}$.

Dethmers et al. mostraram que anastomoses término-terminais ou com enxertos arteriais são superiores àquelas que utilizam enxerto venoso, a patência ou não da anastomose não se relaciona fidedignamente com melhora clínica do paciente; e que, a simpatectomia torácica se relaciona a efeitos colaterais desagradáveis para a maioria dos pacientes ${ }^{7}$. Caetano e Aguiar relatam casos de lesões do nervo ulnar no canal de Guyon por alterações da artéria da artéria alunar e recomendam o tratamento cirúrgico como padrão, com alívio dos sintomas no seguimento a curto prazo ${ }^{2,8}$.

\section{CASO CLÍNICO}

Paciente do gênero masculino, 32 anos, não fumante, trabalhador agrícola, destro, encaminhado ao ambulatório de Cirurgia da Mão e Microcirurgia do HCFMRP-USP com queixa de alterações de sensibilidade no quarto e no quinto dedos da mão direita. Informou o surgimento de pequena nodulação em região hipotenar há seis meses, sem história de trauma prévio. Ao exame físico foi encontrado nódulo não pulsátil, de aproximadamente $1,5 \mathrm{~cm}$ em eminência hipotenar, sem dor ao toque, acompanhada de parestesia do quarto (rebordo ulnar) e quinto dedos. A ressonância nuclear magnética (RNM) evidenciou nodulação superficial ao canal de Guyon (Figura 1). Sem alterações na eletroneuromiografia. Optado por ressecção cirúrgica da tumoração.

Durante o ato operatório, após a abertura do canal e evidenciou-se importante dilatação da artéria ulnar (Figura 2A) que media $1,5 \times 1,0 \times 1,0 \mathrm{~cm}$ e comprimia o nervo ulnar, na zona III do canal de Guyon. Procedeu-se a ressecção do segmento lesionado (Figura 2B), com encaminhamento da peça para o anatomopatológico (Figura 2C). A reconstrução foi realizada por anastomose término- 
terminal por técnica microcirúrgica (Figura 3). O paciente evoluiu com remissão completa do quadro clínico e normalização ao exame físico.

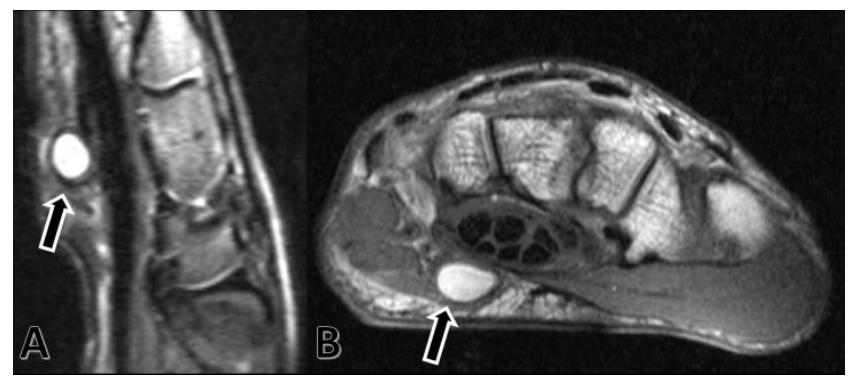

Figura 1: A (corte sagital) e B (corte axial): RNM evidenciando aneurisma (seta).

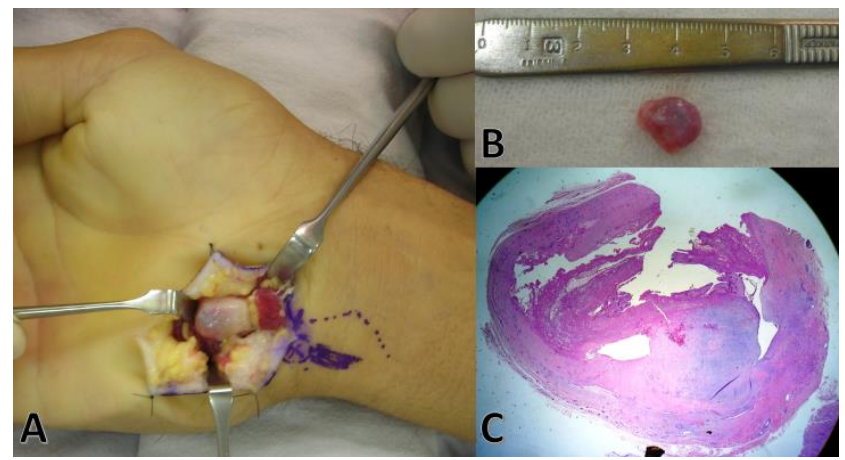

Figura 2: A: aspecto do aneurisma durante exploração. B: Após ressecção. C: Aspecto histológico da lesão com trombo no seu interior (seta) e dilatação do lúmen.

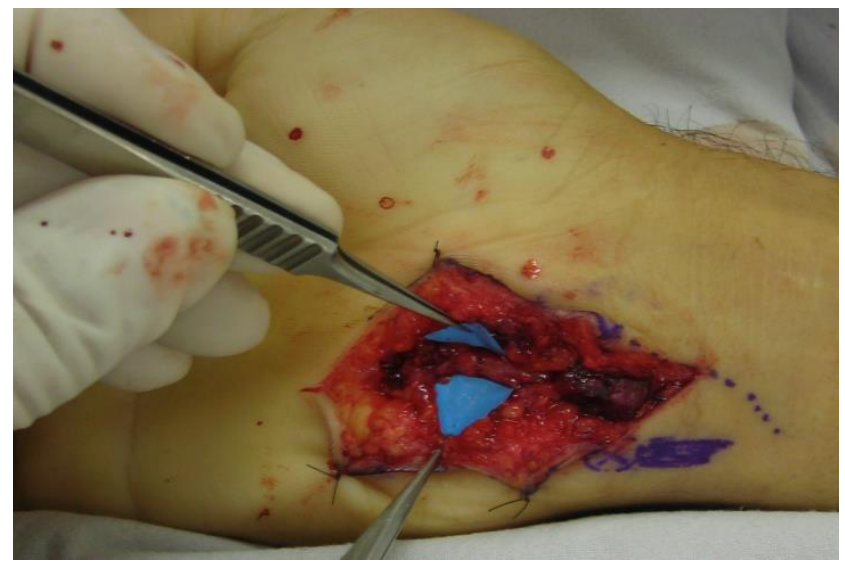

Figura 3: Artéria ulnar após ressecção do aneurisma e reparo.

\section{DISCUSSÃO}

A compressão do nervo ulnar ao nível do canal de Guyon é secundária à compressão por aneurismas verdadeiros é um fenômeno raro. Apesar dos poucos casos disponíveis, seu diagnóstico depende do sítio de compressão, como proposto por Shea-Maclain, e da magnitude dos sintomas. Distúrbios da sensibilidade no $5^{\circ}$ dedo e na borda ulnar do $4^{\circ}$ dedo são comuns, bem como queixas motores. Alguns casos podem apresentar queixas de dor e isquemia, como observado na síndrome do martelo hipotênar ${ }^{7-10}$.

Os aneurismas podem ser do tipo saculares (falsos), decorrentes de trauma penetrante na parede do vaso, não apresentando tecido muscular ou elástico, pois sua parede é composta por tecido fibroso. Ou podem ser do tipo fusiformes (verdadeiros), secundários à traumas repetitivos ou episódio único não-penetrante, e envolvem todas as três camadas da parede vascular ${ }^{6,10}$.

O estudo eletrodiagnóstico pode desempenhar um papel bem definido quanto à localização da lesão compressiva durante a investigação pré-operatória9. A RNM também possui importante papel na determinação da natureza da lesão, no mapeamento do canal de Guyon e, conforme estudos recentes pode, inclusive, revelar a compressão nervosa $^{4-10}$.

Clinicamente encontra-se massa palpável no local da lesão, que pode ser pulsátil em até metade dos casos. Dor local e alterações vasculares, muitas vezes secundárias a processo trombótico manifestamse como eventos isquêmicos, como diminuição da temperatura local e a cianose. Os exames complementares empregados para a investigação de aneurismas são a ultrassonografia Doppler e a arteriografia, sendo esta, útil para o planejamento cirúrgico $^{10}$.

As opções de tratamento incluem a ressecção do aneurisma com reconstrução através de anastomose término-terminal com ou sem interposição de enxerto de veia, a depender do comprimento da falha residual ${ }^{5-10}$.

\section{CONSIDERAÇÕES FINAIS}

Ressecção e ligadura são alternativas encontradas em vários relatos da literatura. Porém, acredita-se que os métodos de reconstrução são mais aconselháveis pela literatura $^{5-10}$. No presente caso, não houve necessidade de enxerto de interposição, e o paciente evoluiu com melhora dos sintomas.

\section{REFERÊNCIAS}

1. Guyon F. Note sur une disposition anatomique proper à la face antérieure de la région du poignet et non encour décrite. Bulletins de la Societé anatomique de Paris, Second Series, 1861;6:184-86.

2. Caetano EB, Brandi S, Oliveira Junior WM. Compressão do nervo ulnar no canal de Guyon por aneurisma verdadeiro: relato de um caso. Rev Bras Ortop. 2004;39(7):398-400.

3. Shea JD, McClain EJ. Ulnar-nerve compression syndromes at and below the wrist. J Bone Joint Surg Am. 1969;51(6):1095-103.

4. Rothkopf DM, Bryan DJ, Cuadros CL, May JW Jr. Surgical management of ulnar artery aneurysms. J Hand Surg Am. 1990;15(6):891-97.

5. Harris EJ Jr, Taylor LM Jr, Edwards JM, Mills JL, Porter JM. Surgical treatment of distal ulnar artery aneurysm. Am J Surg. 1990;159(5):527-30.

6. Dethmers RS, Houpt P. Surgical management of hypothenar and thenar hammer syndromes: a retrospective study of 31 instances in 28 patients. J Hand Surg Br. 2005;30(4):419-23. 
7. Aguiar PH, Bor-Seng-Shu E, Gomes-Pinto F, Almeida- Leme RJ, Freitas ABR, Martins RS et al. Surgical management of Guyon's canal syndrome, an ulnar nerve entrapment at the wrist: report of two cases. Arq Neuro-Psiquiatr. 2001; 59(1):106-11.

8. Jose RM, Bragg T, Srivastava S. Ulnar nerve compression in Guyon's canal in the presence of a tortuous ulnar artery. J Hand Surg Br. 2006; 31(2):200-2.

9. Galati G, Cosenza UM, Sammartino F, Benvenuto E, Caporale A. True aneurysm of the ulnar artery in a soccer goalkeeper: a case report and surgical considerations. Am J Sports Med. 2003; 31(3):457-58.

10. Rothkopf DM, Bryan DJ, Cuadros CL, May JW Jr. Surgical management of ulnar artery aneurysms. J Hand Surg Am. 1990;15(6):891-97.

\section{CONFLITO DE INTERESSES}

Os autores declaram não haver conflitos de interesse.

\section{AUTOR PARA CORRESPONDÊNCIA}

Msc. Dr. Luis Guilherme Rosifini Alves Rezende

Hospital das Clínicas da Faculdade de Medicina de Ribeirão Preto da Universidade de São Paulo (HC-FMRP-USP)

Rua Tenente Catão Roxo, 3900 - Vila Monte Alegre 14015-010 Ribeirão Preto - SP, Brasil

Telefone: (16) 98149-5454

E-mail: lgrarezende@hcrp.usp.br

Submetido em 15/12/2020

Aceito em 23/12/2020 